\title{
Micro-ribonucleic acid 29b inhibits cell proliferation and invasion and enhances cell apoptosis and chemotherapy effects of cisplatin via targeting of DNMT3b and AKT3 in prostate cancer
}

\author{
This article was published in the following Dove Press journal: \\ OncoTargets and Therapy \\ 4 March 2015 \\ Number of times this article has been viewed
}

Bin Yan'

Qiong Guo ${ }^{2}$

Xiao-xin $\mathrm{Nan}^{2}$

Zhao Wang'

Zhuo Yin'

Lu Yi'

Yong-bao Wei'

Yun-liang Gao'

Ke-qin Zhou'

Jin-rui Yang'

'Department of Urology, Second Xiangya Hospital, Central South

University, ${ }^{2}$ Department of Urology,

Third Changsha Hospital, Changsha,

Hunan, People's Republic of China
Correspondence: Jin-rui Yang

Department of Urology, Second Xiangya Hospital, Central South University,

Renmin road no. 139, Changsha, Hunan

4I 00 I I, People's Republic of China

Tel +86 I360744 3048

Email yangjr06@163.com

\section{Qiong Guo}

Department of Urology, Third Changsha Hospital, Labor road no. 176, Changsha, Hunan 4I00I5, People's Republic of China

Tel +86 I387596 660।

Email guoqiong06@I63.com
Background: Micro-ribonucleic acids (miRNAs) are crucial regulators in malignant tumors. miRNA-29b (miR-29b) has been identified as a tumor suppressor in prostate cancer (PCa). However, very few studies have investigated the effects of miR-29b in PCa, especially the mechanism and its association with chemotherapy. Our study aimed to explore the role and mechanism of miR-29b in PCa.

Materials and methods: The expression levels of miR-29b were detected in ten clinical PCa specimens and four different $\mathrm{PCa}$ cell lines through quantitative real-time polymerase chain reaction. After miR-29b mimics and inhibitors were successfully transfected into LNCaP, the 3-(4,5-dimethylthiazol-2-yl)-2,5-diphenyltetrazolium bromide assay was then used to investigate cell proliferation and cisplatin sensitivity of PCa cells. Cell cycle, cell apoptosis, and cell invasion were detected via flow cytometry, annexin V-fluorescein isothiocyanate labeling, and transwell assay, respectively. Based on bioinformatic methods, Western blot analysis, and dual-luciferase reporter assay, novel target genes of miR-29b were identified.

Results: miR-29b was downregulated in PCa tissues compared with matched adjacent nontumor tissues. In the androgen-independent PCa cell line (LNCaP-AI), the expression of miR-29b was much lower than the androgen-dependent PCa cell line (LNCaP). Subsequent studies showed that forced expression of miR-29b inhibited cell proliferation and cell invasion and induced cell apoptosis in PCa. Upregulation of miR-29b also enhanced the chemosensitivity of PCa cells to cisplatin. Moreover, we identified DNMT3b and AKT3 as novel target genes of miR-29b in PCa.

Conclusion: Taken together, the results showed that miR-29b plays a tumor-suppressive role in PCa. It inhibits cell biological behavior and enhances the chemotherapy effects of cisplatin through its involvement in epigenetic regulation and PI3K/AKT pathway.

Keywords: miRNA-29b, prostate cancer, cell biological behavior, chemosensitivity, DNMT3b, AKT3

\section{Introduction}

Prostate cancer (PCa) is one of the most lethal diseases in men worldwide. It is also the second-leading cause of cancer deaths among men in developed countries. ${ }^{1}$ Chemotherapy is a mainstay therapy for $\mathrm{PCa}$, especially for advanced $\mathrm{PCa}$. However, most PCa patients will develop chemoresistance following a period of chemotherapy. Chemoresistance remains challenging and difficult for PCa researchers. Therefore, finding new approaches to reverse chemoresistance in PCa would be a huge step forward in the treatment of $\mathrm{PCa} .^{2}$ 
Micro-ribonucleic acids (miRNAs) are endogenous small noncoding RNAs. They work as post-transcriptional regulators of gene expression by binding to the $3^{\prime}$-untranslated region (UTR) of target messenger RNAs (mRNAs). ${ }^{3,4}$ One miRNA can bind to a number of mRNA transcripts, and in turn one mRNA can be targeted by a widespread panel of miRNA species. ${ }^{5}$ Evidence has shown that miRNAs are crucial regulators of cancer by targeting different signaling pathways and multiple transcripts. ${ }^{6,7}$

The miRNA (miR)-29 family consists of three members miR-29a, miR-29b, and miR-29c - differing only in two or three bases. ${ }^{8,9}$ As a member of miR-29 family, miR-29b is generally recognized as a fundamental regulator of the epithelial-mesenchymal transition (EMT) involved in cancer metastasis and chemoresistance. ${ }^{10,11}$ Several reports have revealed that miR-29b is a critical regulator of cancer, mostly through targeting oncogenes and exerting epigenetic regulation. Studies have reported that miR-29b is downregulated in $\mathrm{PCa}$ and acts as a tumor suppressor. ${ }^{10}$ However, there are very few studies focusing on the role and underlying mechanisms of miR-29b in PCa. Based on these facts, we undertook this study to investigate the specific roles of miR-29b in PCa. Its effects on cell biological properties, chemoresistance, and target genes in PCa were explored.

\section{Materials and methods Clinical prostate specimens}

Clinical specimens were obtained from patients at the Second Xiangya Hospital of Central South University, Changsha. All the patients had been diagnosed with PCa and had undergone radical prostatectomy during April 2012 to October 2013. Cancer tissues and matched noncancerous tissues were obtained immediately after the prostates were resected. The specimens were then maintained at $-80^{\circ} \mathrm{C}$. The protocol was approved by the Institutional Review Board of Central South University.

\section{Cell lines and cell culture}

The human PCa cell lines PC3, DU145, and LNCaP were procured from the cell bank of the Chinese Academy of Sciences in Beijing, People's Republic of China (PRC) and were cultured in Roswell Park Memorial Institute (RPMI) 1640 medium containing $10 \%$ fetal bovine serum (FBS), $100 \mathrm{U} / \mathrm{mL}$ penicillin, and $100 \mu \mathrm{g} / \mathrm{mL}$ streptomycin (Sigma-Aldrich, St Louis, MO, USA) in a humidified incubator at $37^{\circ} \mathrm{C}$ containing $5 \% \mathrm{CO}_{2}$. The androgen-independent cell line LNCaP-AI was derived from $\mathrm{LNCaP}$ after culturing in androgen-removed culture medium. The LNCaP-AI cell line was cultured in
RPMI 1640 medium with FBS after processing with 10\% activated carbon and glucose. A miR-29b mimic sequence was synthesized by GenePharma Technology (Shanghai, PRC). The miR-29b mimic sequence was transfected at working concentrations using Lipofectamine 2000 reagent (Thermo Fisher Scientific, Waltham, MA, USA).

\section{Quantitative real-time PCR}

Total RNA was extracted using Trizol reagent from the cells (Trizol RNA-extraction kit; Qiagen, Venlo, the Netherlands) according to the manufacturer's protocol. For miRNAexpression analysis, complementary deoxyribonucleic acid (DNA) was synthesized from total RNA (10 ng) using a TaqMan miRNA reverse-transcription kit (Thermo Fisher Scientific) with miRNA-specific primers. RNU6 was used as an internal control. The primer of miRNA amplification was miR-29b-forward 5'-GGGTAGCACCATTTGAAATC-3', miR-29b-reverse 5'-TTTGGCACTAGCACATT-3', U6forward 5'-CTCGCTTCGGCAGCACA-3', and U6-reverse 5'-AACGCTTCACGAATTTGCGT-3'. Quantitative realtime polymerase chain reaction (qRT-PCR) was carried out using All-in-One ${ }^{\mathrm{TM}}$ q-PCR Mix (GeneCopoeia, Rockville, MD, USA) on an ABI Prism 7900HT system (Thermo Fisher Scientific). Template-negative and reverse transcriptionnegative conditions were used as controls. All experiments were repeated three times, and all assays contained a negative-control reaction group. All samples were normalized to internal controls. The fold change in expression was then obtained using the ddCt method.

\section{Transfection with miR-29b mimics}

The hsa-miR-29b mimics and negative-control miRNA were respectively used (hsa-miR-29b mimics sense 5'-UAGCACCAUUUGAAAUCAGUGUU-3', antisense 5'-AACACUGAUUUCAAAUGGUGCUA-3'; negative-control miRNA sense 5'-UUCUCCGAACGUGUCACGUTT-3', antisense 5'-ACGUGACACGUUCGGAGAATT-3'). LNCaP cells were seeded in six-well plates and cultured with serum-free medium for 24 hours. Lipofectamine 2000 was used to transfect cells with miR-29b mimics, inhibitors, or negative-control miRNA according to the instructions. Concentrations for miR-29b mimics and inhibitors were $2 \mu \mathrm{M}$. RPMI 1640 containing $10 \%$ FBS was used to culture transfected cells. miR-29b mimics and negative-control miRNA were synthesized by GenePharma Technology. The transfection efficiencies of miRNA in LNCaP cells were confirmed based on the expression level of miR-29b by quantitative real-time (qRT)-PCR-PCR and immunofluorescent assay. Transfection efficiency more than $80 \%$ was defined as 
successful transfection. Fluorescent cells and normal cells were observed under light microscopy and fluoroscopy, respectively. Transfection efficiency in our experiment was more than $80 \%$, indicating a successful transfection (Figure 1A). Relative miR$29 \mathrm{~b}$ expression levels in the transfection group were significant higher than the negative-control group ( $P<0.05$, Figure 1B).

\section{Cell proliferation and cisplatin sensitivity (MTT assay)}

Based on the level of miR-29b expression, the LNCaP cell line was selected for the research. Cisplatin was obtained from Selleck Chemicals (Shanghai, PRC). According to the results of our preliminary experiment, the optimum cisplatin concentration was $5.0 \mu \mathrm{g} / \mathrm{mL}$. LNCaP cells of each group were plated in 96 -well plates at $1 \times 10^{4}$ per well in a final volume of $100 \mu \mathrm{L}$. 3-(4,5-Dimethylthiazol-2-yl)-2,5diphenyltetrazolium bromide (MTT) was added after incubation for 24 hours in a humidified incubator at $37^{\circ} \mathrm{C}$ with $5 \%$ $\mathrm{CO}_{2}$. The plates were incubated for another 4 hours. Dimethyl sulfoxide (Sigma-Aldrich) was used at $37^{\circ} \mathrm{C}$ for 10 minutes following removal of culture medium. The absorbance was measured at $570 \mathrm{~nm}$ by a microplate reader (Molecular Devices, Sunnyvale, CA, USA).

\section{Cell-cycle, cell-apoptosis, and cell- invasion assays}

To evaluate the effect of miR-29b on the cell cycle and cell apoptosis, flow cytometry and annexin $\mathrm{V}$-fluorescein isothiocyanate (FITC) labeling was used. The cells transfected with miR-29b mimics or negative-control miRNA were harvested and resuspended in $100 \mathrm{~mL}$ of flow-cytometry staining buffer. The buffer contained $1 \times$ phosphate-buffered saline (PBS) and $2 \%$ FBS. The cells were then stained with propidium iodide (PI; Sigma-Aldrich) staining solution. PI fluorescence was measured, and PI fluorescence-positive cells were counted as dead cells. The collected cells were resuspended in $0.5 \mathrm{~mL}$ of PBS. Then, they were fixed with $4.5 \mathrm{~mL}$ of $70 \%$ ethanol for 4 hours, washed with PBS, and stained with PI staining solution. The cell-cycle distribution was determined by using a FACSCalibur flow cytometer (FL-2 channel). Cell apoptosis was detected via an annexin V-FITC labeling kit purchased from Kaiji Biotech (Nanjing, PRC), and was carried out
A

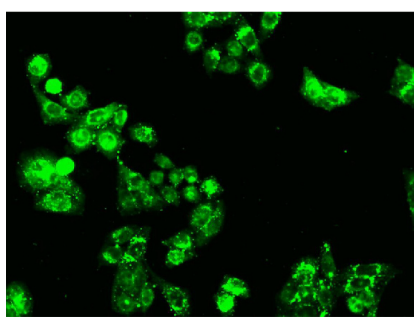

Image in fluoroscopy

C

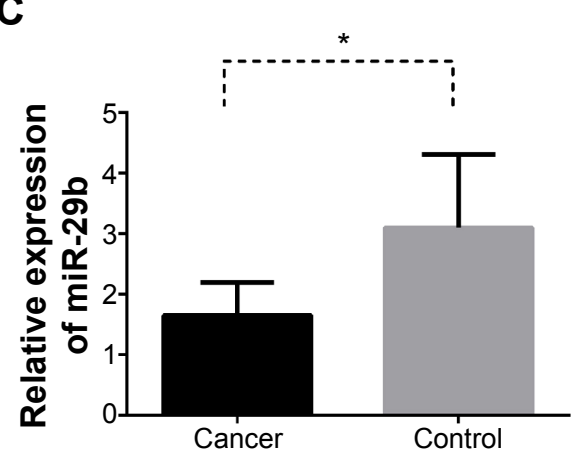

B
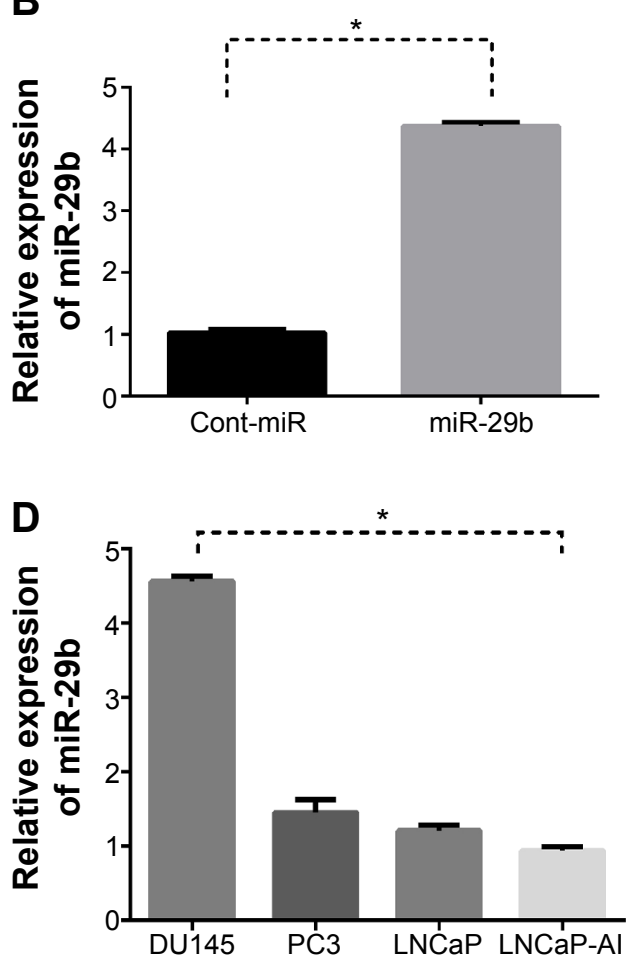

Figure I miR-29b is downregulated in prostate cancer.

Notes: (A) Transfection efficiency of miR-29b mimics was evaluated, and more than $80 \%$ of cells were found to be transfected in light microscopy and fluoroscopy, suggesting the transfection was successful. (B) Relative miR-29b expression in the miR-29b mimic group (miR-29b group) was significantly higher than the negative-control miR (ContmiR) group $(* P<0.05)$. (C) Comparison of miR-29b expression in prostate cancer tissues (Cancer) and compared with patient-matched control noncancerous prostate tissues (Control). miR-29b expression was significantly lower in the cancer group $(* P<0.05)$. (D) Comparison of miR-29b expression in different prostate cancer cell lines. miR-29b expression was significantly lower in LNCaP-Al cell lines $(* P<0.05)$.

Abbreviation: miR, micro-ribonucleic acid. 
according to the supplier's instructions. FITC-labeled cells were counted and analyzed by a FACSAria ${ }^{\mathrm{TM}}$ flow cytometer (BD Biosciences, San Jose, CA, USA).

Matrigel (BD Biosciences) was utilized according to the instructions. Diluted with FBS-free RPMI 1640 with a ratio of 1:8, the diluted Matrigel was then added to the transwell bottom chamber. $\mathrm{LNCaP}$ cells were treated with $0.25 \%$ trypsin and added to RPMI 1640 to prepare a $1 \times 106 / \mathrm{mL}$ single-cell suspension. A transwell chamber was then put into a 24-well plate. RPMI $1640(600 \mu \mathrm{L})$ containing 10\% FBS and $200 \mu \mathrm{L}$ of the prepared single-cell suspension were added. After 48 hours' incubation at $37^{\circ} \mathrm{C}$ in an incubator with $5 \% \mathrm{CO}_{2}$, all of the liquid was removed from the transwell chamber and the bottom chamber. Nitrocellulose membranes were used for Western blot analysis. The membrane was then washed with PBS three times, immersed in methanol, and kept for 20 minutes at room temperature, followed by hematoxylin staining for 10 minutes. The cells that migrated through the pores to the lower surface of the membrane were counted under a microscope $(\times 400)$.

\section{Bioinformatics and Western blot analysis}

Identification of potential miR-29b target genes was performed using the TargetScan and PicTar databases. The putative miRNA targets included the most recent TargetScan predictions and the $3^{\prime}$-UTR targets reported by $\mathrm{Ru}$ et al. ${ }^{10}$ Protein lysates were separated by $10 \%$ sodium dodecyl sulfate polyacrylamide gel electrophoresis (Sigma-Aldrich) and transferred to nitrocellulose blotting membranes (Pierce, Rockford, IL, USA). The membranes were then incubated with fluorescence-labeled secondary antibody IgG (1:4,000 dilution). GAPDH was used as the control. The company information and catalog numbers for antibodies SOCS-1, Mcl-1, DNMT3b, MMP2, IFN- $\gamma$, SMAD3, SOCS5, AKT3, and GAPDH were as follows: ABCAM/ab9870, Cst/5453, Abcam/ab2851, Abcam/ab124294, Cst/3159, Cst/9523, Abcam/ab3695, Cst/8018, and SANTA/SC-365062, respectively. After exposure and development, protein expression was analyzed using a gel-imaging analysis system (Gel-Pro 4.0). Western blot assays were performed in triplicate.

\section{Dual-luciferase reporter assay}

The Dual-Luciferase ${ }^{\circledR}$ Reporter Assay System (Promega, Fitchburg, WI, USA) was used according to the protocol. Dual-luciferase reporter constructs containing the putative miR-29b binding sites from DNMT3b and AKT3 were used as described previously. ${ }^{12,13}$ Briefly, partial sequences of the $3^{\prime}$-UTR of AKT3 (454 nt) and sequences of the 3 '-UTR of DNMT3b (443 nt) were inserted in the pmirGLO vector
(Promega). Constructs were cotransfected into LNCaP cells cultured in 12-well plates using Lipofectamine 2000. Briefly, $30 \mathrm{nM}$ miRNA mimics, inhibitors, or control miRNA was transfected with $0.6 \mu \mathrm{g}$ of the pMiR 3'-UTR clones. Luciferase activity was measured at 48 hours posttransfection.

\section{Statistical analysis}

SPSS 16.0 software was used for statistical analysis. All data are presented as means \pm standard deviation of three independent experiments. Student's two-tailed $t$-test was used for comparisons of two independent groups. $P$-values $<0.05$ were considered statistically significant.

\section{Results \\ Expression levels of miR-29b in prostate cancer specimens and cancer cell lines}

Ten patients receiving radical prostatectomy were included in our study, with approval of the Institutional Review Board of Central South University. All the patients provided written informed consent. The age range of these ten patients was 56-74 years, with an average age of 68.1 years. There were seven T1-T2 patients of and three T3 patients according to TNM (tumor, node, metastasis) staging. There were four G1-G2 patients and six G3-G4 patients of according to pathologic grading (Table 1). miR-29b expression was significantly lower in cancer tissues than in noncancerous tissues $(P<0.05$, Figure 1C). However, no statistical significance and no correlation between miR-29b levels and Gleason grade or TNM stage was found.

The relative expression of miR-29b in four $\mathrm{PCa}$ cell lines (DU145, PC3, LNCaP, and LNCaP-AI) were 4.56 \pm 0.14 , $1.45 \pm 0.36,1.20 \pm 0.08$, and $0.99 \pm 0.13(P<0.05)$, respectively. The expression level of miR-29b in LNCaP-AI cells, which

Table I Patient characteristics

\begin{tabular}{lllllll}
\hline Number & $\begin{array}{l}\text { Age } \\
\text { (years) }\end{array}$ & $\begin{array}{l}\text { PSA } \\
\text { (ng/mL) }\end{array}$ & $\begin{array}{l}\text { Gleason } \\
\text { score }\end{array}$ & \multicolumn{2}{l}{$\begin{array}{l}\text { TNM } \\
\text { classification }\end{array}$} \\
\cline { 5 - 7 } & & & & T & $\mathbf{N}$ & $\mathbf{M}$ \\
\hline 1 & 65 & 48.5 & $4+3$ & 2 & 0 & 0 \\
2 & 56 & 20.3 & $3+3$ & 2 & 0 & 0 \\
3 & 67 & 56.4 & $4+4$ & $3 b$ & 1 & 0 \\
4 & 70 & 26.7 & $3+3$ & 2 & 0 & 0 \\
5 & 74 & 43.7 & $4+5$ & $3 b$ & 1 & 0 \\
6 & 73 & 21.9 & $4+4$ & 2 & 0 & 0 \\
7 & 71 & 19.6 & $3+3$ & 2 & 0 & 0 \\
8 & 72 & 38.9 & $4+3$ & $3 b$ & 0 & 0 \\
9 & 66 & 31.2 & $4+5$ & 2 & 0 & 0 \\
10 & 67 & 17.6 & $3+3$ & 2 & 0 & 0 \\
\hline A & 73 & &
\end{tabular}

Abbreviations: PSA, prostate-specific antigen; TNM, tumor, node, metastasis. 
represented the androgen-independent cell line, was significantly lower than miR-29b expression in LNCaP cells (Figure 1D). Since LNCaP cells showed relatively low miR-29b expression level and had been mostly used in our previous studies, they were selected for our next experiments.

\section{Upregulation of miR-29b expression inhibits cell growth and invasion and induces apoptosis of PCa cells}

Cell-cycle changes were examined by flow cytometry in the miR-29b mimic-transfected group (miR-29b group) compared with the negative-control group (Cont-miR). In the miR-29b group, the proportion of sub- $\mathrm{G}_{0} / \mathrm{G}_{1}$-phase cells increased significantly, and the proportion of S-phase and $\mathrm{G}_{2} / \mathrm{M}$-phase cells decreased significantly compared with the Cont-miR group $(P<0.05$, Figure $2 \mathrm{~A})$, indicating that overexpression of miR-29b inhibited cell growth in $\mathrm{PCa}$.

Cell apoptosis was then determined by annexin V-FITC labeling. The total apoptosis rate in the miR-29b group was significantly higher than in the Cont-miR group $(P<0.05$, Figure 2B), suggesting that overexpression of miR-29b induced cell apoptosis in PCa.

Similar results were revealed in transwell chambers for invasion assay between the miR-29b group and Cont$\mathrm{miR}$ group. The invasion cells were more significantly reduced in the miR-29b-transfected group compared to the Cont-miR group $(P<0.05$, Figure $2 \mathrm{C})$, demonstrating that increased miR-29b expression repressed cell invasion in PCa.
A
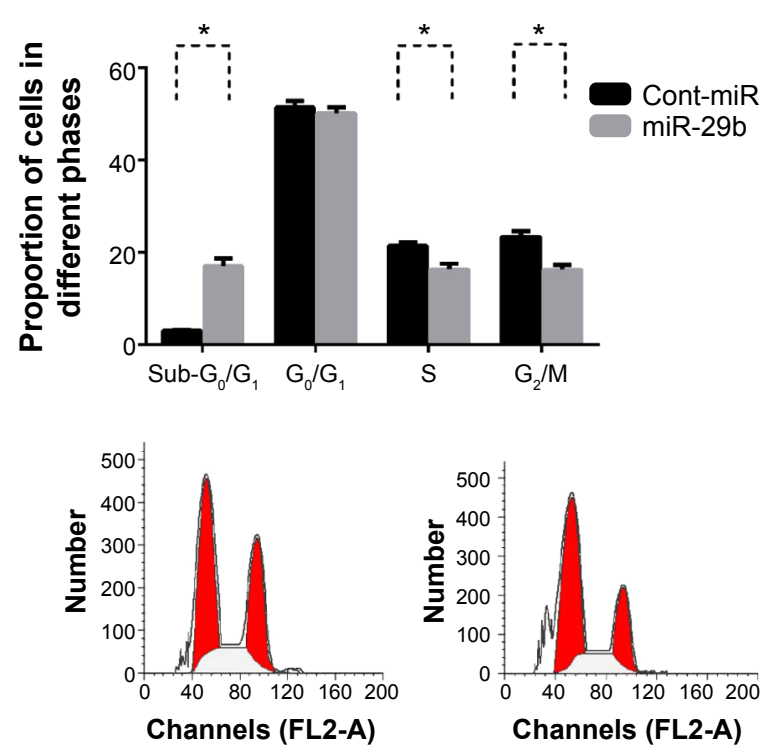

Cont-miR

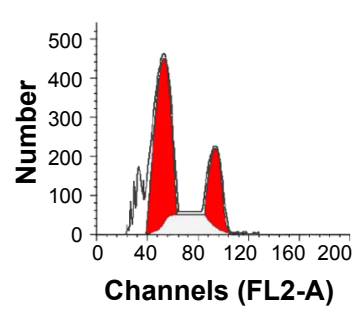

miR-29b

C

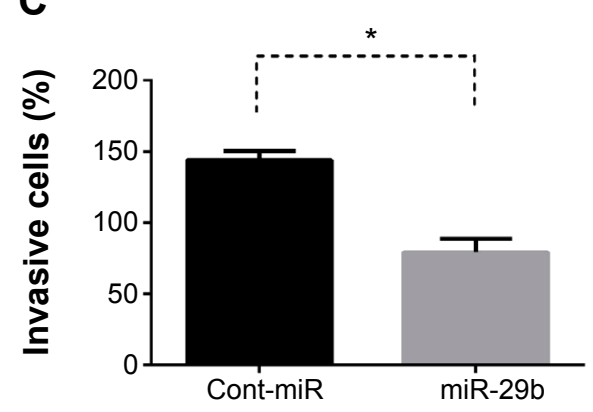

B
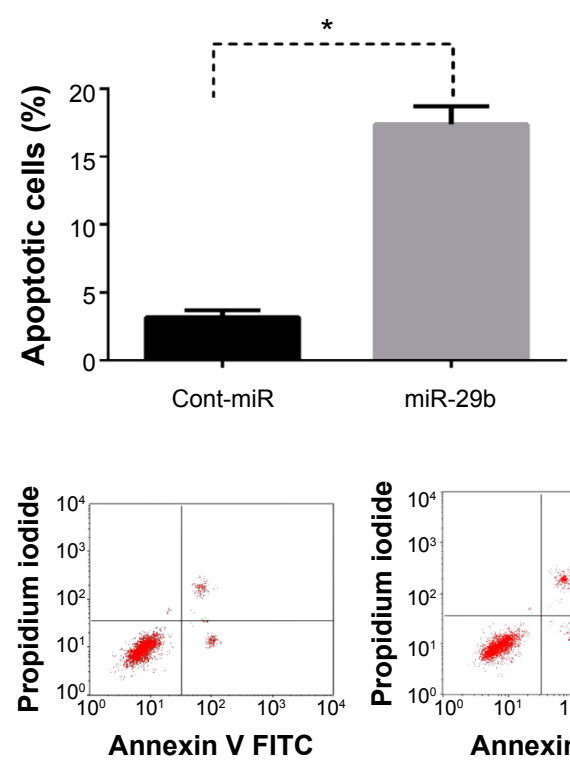

Cont-miR

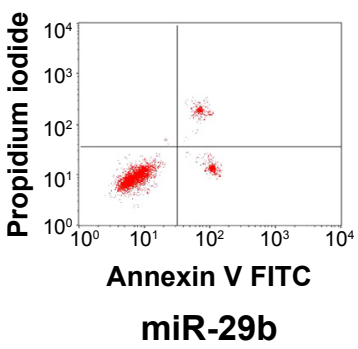

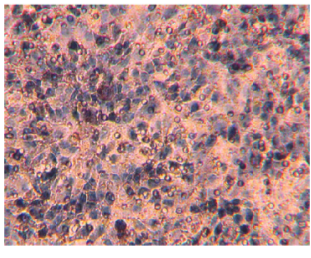

Cont-miR

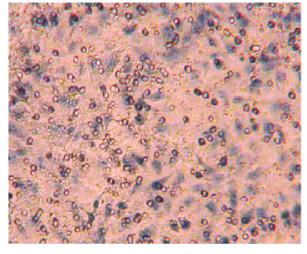

miR-29b

Figure 2 Upregulation of miR-29b expression inhibited growth and invasion and induced apoptosis of prostate cancer cells.

Notes: (A) In the miR-29b mimic-transfected group (miR-29b), the proportion of sub-G/G -phase cells increased significantly, and the proportion of S-phase and $\mathrm{G}_{2} / \mathrm{M}_{0}-\mathrm{phase}$ cells decreased significantly compared with the negative-control group (Cont-miR). (B) The percentage of apoptotic cells in the miR-29b group was significantly higher than in the Cont-miR group $(* P<0.05)$. (C) The invasion cells were significantly reduced in the miR-29b-transfected group compared to the negative-control group $(* P<0.05)$. Abbreviations: miR, micro-ribonucleic acid; FITC, fluorescein isothiocyanate. 


\section{Overexpression of miR-29b enhances} chemosensitivity of PCa cells to cisplatin

There were five groups: the blank control group (Blank), LNCaP transfected with negative-control miRNA (ContmiR), LNCaP transfected with negative-control miRNA plus cisplatin (Cont-miR + cisplatin), LNCaP transfected with miR-29b mimics (miR-29b), and LNCaP transfected with miR-29b mimics plus cisplatin (miR-29b + cisplatin). The LNCaP cell-growth rate in miR-29b was significant lower than in Blank and Cont-miR $(P<0.05)$, indicating that miR-29b inhibited cell proliferation of PCa. The cell-growth rate in miR-29b + cisplatin was significantly lower than Cont-miR + cisplatin and miR-29b $(P<0.05)$, suggesting that overexpression of miR-29b enhanced the chemotherapy of cisplatin on $\mathrm{PCa}$ (Figure 3A).

\section{miR-29b directly targets DNMT3b and AKT3}

Potential miR-29b target genes were searched using the TargetScan and PicTar databases. Eight potential candidate target genes were screened, including SOCS-1, Mcl-1, DNMT3b, $M M P 2, I F N \gamma$, SMAD3, SOCS5, and AKT3. In parallel, $G A P D H$ was also included as a control. Western blot analysis was then done to detect the protein-expression levels of these nine genes (Figure 3B). Among these, the protein expression of $M c l-1, D N M T 3 b$, and $A K T 3$ was significantly decreased in the miR-29b mimic-transfection group and increased in the miR-29b inhibitor-transfection group (Figure 3C). Since previous research by Steele et al has demonstrated that $\mathrm{Mcl}-1$ is a target gene in $\mathrm{PCa}$, we focused on the genes of $D N M T 3 b$ and $A K T 3 .{ }^{12}$ We found putative miR-29b target sites in the DNMT3b 3'-UTR and AKT3 3'-UTR (Figure 4A). Dual-luciferase reporter assays showed reduced luciferase activities in miR-29b mimic-transfected cells and increased luciferase activities in miR-29b inhibitor-transfected cells in both the DNMT3b (Figure 4B) and AKT3 groups (Figure 4C). The results demonstrated that miR-29b targets DNMT3b and AKT3 directly.

\section{Discussion}

miRNAs have been known to affect cellular biological behaviors, including the cell cycle and cell proliferation, apoptosis, and invasion. ${ }^{5}$ There are huge associations of miRNAs with tumor etiology, progression, and prognosis in multiple human cancers. ${ }^{6}$ Various studies have demonstrated that miR-29b acts as a tumor-suppressive miRNA in many different cancers. However, relatively little research has focused on the effects of miR-29b on PCa. Ru et al demonstrated that
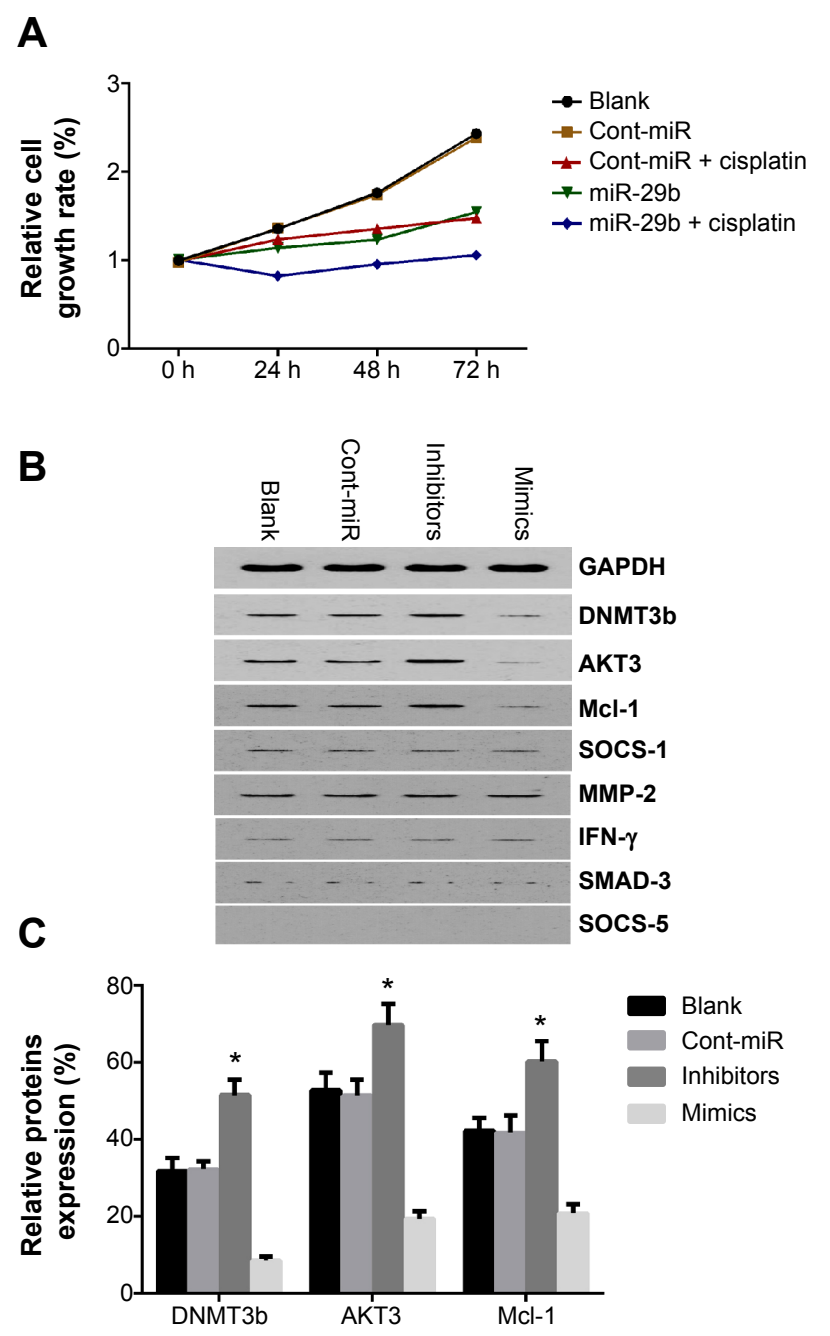

Figure 3 Overexpression of miR-29b enhances chemosensitivity of cisplatin on prostate cancer cells. miR-29b targets DNMT3b and AKT3.

Notes: (A) Compared with the blank-control (blank) and negative-control miR (Cont-miR) groups, the relative cell-growth rate in the miR-29b group was significantly lower along with action time, indicating that miR-29b inhibited cell proliferation of prostate cancer. Cell viability in the miR-29b group plus cisplatin was significantly lower than the miR plus cisplatin-control group and the miR-29b group, suggesting that overexpression of miR-29b enhanced the chemotherapy of cisplatin for prostate cancer. (B) Western blotting of eight potential genes' expression at the protein level in the blank, negative-control miRNA, miR-29b inhibitor-transfection, and miR-29b mimic-transfection groups. (C) The quantitative protein expression of DNMT3b, AKT3, and Mcl-I were significantly increased in the miR-29b mimictransfection group (Mimics) and decreased in the miR-29b inhibitor-transfection group (Inhibitors). $* P<0.05$.

Abbreviation: miR, micro-ribonucleic acid.

miR-29b can inhibit cell growth by targeting antiapoptotic and prometastatic molecules in PCa. miR-29b also acts as an antimetastatic miRNA in PCa cells by regulating EMT signaling. ${ }^{10,12}$

In our study, we have illustrated that miR-29b expression is decreased in PCa tissues compared with noncancerous tissues, in accordance with previous research results. ${ }^{10}$ However, no statistical significance and no correlation between levels of miR-29b and Gleason grade or TNM stage 
A

DNMT3b 5' -UUUUACUCUUCUUACUGGUGCUA...

hsa-miR-29b 3' -UUGUGACUAAAGUUUACCACGAU

AKT3 5'-UCAGAUUAAACCCUUUGGUGCUA

|| ||$\quad|||||| \mid$

hsa-miR-29b 3' -UUGUGACUAAAGUUU-----ACCACGAU
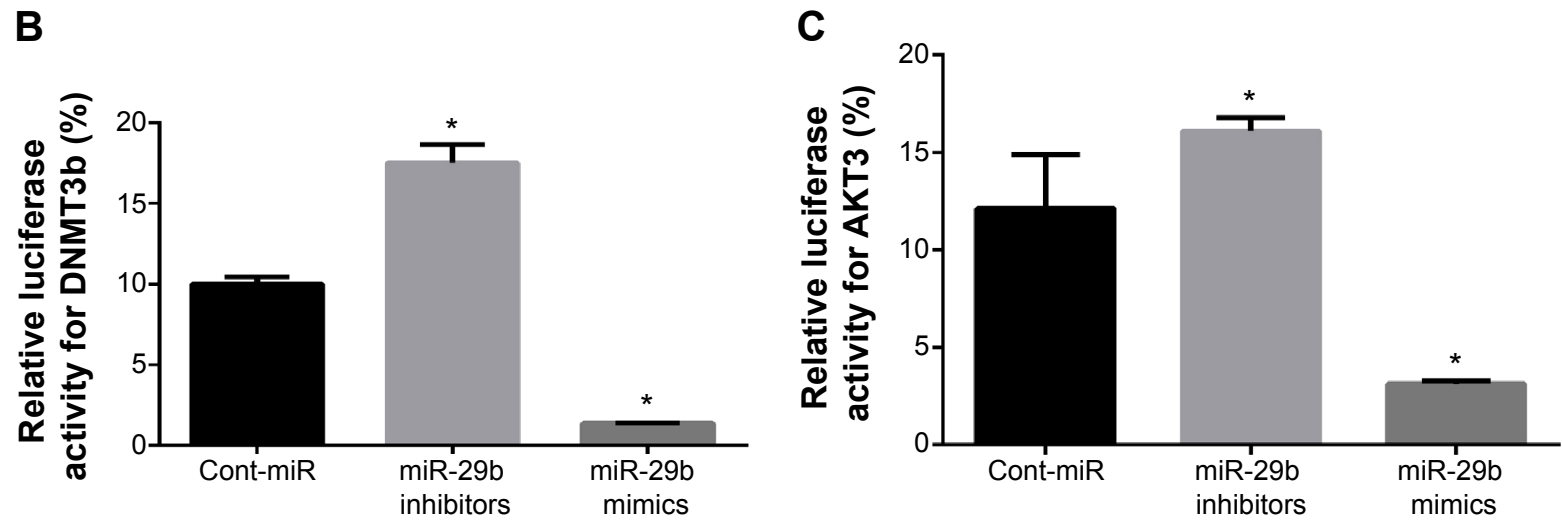

Figure 4 miR-29b directly targets DNMT3b and AKT3.

Notes: (A) Computational analysis of 3'UTR of DNMT3b and AKT3 revealed the 3'-UTR-binding sites. The miR-29b target regions of DNMT3b and AKT3 are indicated. (B) Luciferase assay showing increased reporter activity after cotransfection of DNMT3b with miR-29b inhibitors and decreased reporter activity after cotransfection of DNMT3b with miR-29b mimics $(* P<0.05)$. (C) Luciferase assay showing increased reporter activity after cotransfection of AKT3 with miR-29b inhibitors and decreased reporter activity after cotransfection of AKT3 with miR-29b mimics $(* P<0.05)$

Abbreviations: UTR, untranslated region; miR, micro-ribonucleic acid.

was found. In PCa cells, miR-29b expression was lower in androgen-independent cells than in androgen-dependent cells. This might indicate the association of miR-29b with castration-resistant PCa (CRPC) progress. To assess miR29b's effects on cellular biological behavior, miR-29b mimics were transfected into PCa cells, and the cell cycle, proliferation, apoptosis, and invasion were then detected. Our findings showed that forced expression of miR-29b inhibited cell proliferation and cell invasion and induced cell apoptosis in PCa. Moreover, miR-29b upregulation enhanced the chemosensitivity of cisplatin on $\mathrm{PCa}$, implying a potential role in solving the issue of chemoresistance in $\mathrm{PCa}$.

With regard to how miR-29b potentiates the effects of enhancing chemosensitivity of PCa cells, there are some possible mechanisms. In acute myeloid leukemia, the epigenetic regulation of miR-29b can enhance the chemotherapy effects. ${ }^{13}$ Probably, miR-29b could also increase the chemosensitivity of $\mathrm{PCa}$ through epigenetic regulation. Moreover, EMT is involved in the resistance of cancer cells to chemotherapy, and miR-29b is generally recognized as a fundamental regulator of EMT involved in cancer metastasis and chemoresistance. ${ }^{12,14}$ miR-29b could also increase the chemosensitivity of PCa cells to cisplatin through targeting
EMT-related factors. What is more, studies have shown that the PI3K/AKT pathway is involved in the mechanism of cisplatin effects in various cancers. ${ }^{15,16} \mathrm{miR}-29 \mathrm{~b}$ has been identified as a negative regulator of the PI3K/AKT pathway. ${ }^{17}$ Our findings demonstrated that AKT3 is a target gene of miR-29b in $\mathrm{PCa}$. As a result, miR-29b may potentiate its effects of $\mathrm{PCa}$ chemosensitivity to cisplatin through regulating the PI3K/AKT pathway. In summary, miR-29b could regulate cancer chemosensitivity, possibly through exerting epigenetic effects and regulating the EMT process and PI3K/AKT pathway.

Previous studies on PCa have identified Mcl-1, COL1A1, COL4A1, and MMP-2 as target genes of miR-29b. ${ }^{12}$ Subsequent research indicated that miR-29b directly targets Snail, a key gene involved in the metastatic phenotype. ${ }^{10}$ These results partly shed light on how miR-29b exerts tumor-suppressor effects on PCa. To further investigate possible functional mechanisms of miR-29b in PCa and to identify more target genes of miR-29b, we used bioinformatic methods and Western blot to screen potential candidate target genes. Dualluciferase reporter assays were then performed to identify target genes, since $\mathrm{Mcl}-\mathrm{l}$ has been identified as a target gene of miR-29b multiple myeloma as well as PCa. ${ }^{12,18}$ Therefore, 
we focused on DNMT3 $b$ and $A K T 3$ to detect if these two genes were novel target genes of miR-29b in PCa. In this article, the results of dual-luciferase reporter assays identified that miR-29b targeted DNMT3b and AKT3 directly.

Epigenetic regulations are alterations in gene expression, including DNA methylation, histone modification, and RNA-associated silencing, which are very important pathways in modulating malignant cells. DNA methylation can affect specific gene expression and result in tumor occurrence or antitumor activity. ${ }^{19}$ DNMT3b is a member of the DNA methyltransferase family, which generally exerts DNA-methylation effects, accounting for inactivation of tumor-suppressor genes in many cancer cells. Various studies have shown that miR-29b can target DNA methyltransferase and downregulate the global DNA methylation in malignant cells, through which miR-29b exerts its tumor-suppressive role. Increased miR-29b expression could lead to marked downregulation of the expression of DNA methyltransferases, including DNMT1, DNMT3a, and DNMT3b, by direct targeting or indirect interaction in several cancers. ${ }^{20-22}$ In PCa, hypermethylation of tumorsuppressor genes has been found to be associated with advanced PCa. DNMT activity and DNMT levels were higher in PCa cell lines compared to their nonneoplastic cells, and DNMT3b activity was significantly higher in more aggressive PCa cell lines. ${ }^{23}$ Moreover, DNMT inhibitors could be potential therapeutic agents for advanced PCa. ${ }^{24}$ In this study, we observed that forced expression of miR-29b inhibited DNMT3b protein levels by directly targeting DNMT3b, consistent with previous studies in other tumors. This result indicates that epigenetic regulation of miR-29b is a very important pathway of tumor suppression in $\mathrm{PCa}$.

Activation of the PI3K pathway, resulting from loss of the PTEN tumor-suppressor gene, is one of the predominant pathways in $\mathrm{PCa} .{ }^{25}$ The serine/threonine-kinase AKT family is the primary downstream mediator of PI3K signaling. The AKT family consists of three members - AKT1, AKT2, and AKT3 - that are highly homologous isoforms. Although sharing a high degree of homology, the AKT family shows distinct roles in human cancers. ${ }^{26,27}$ In triple-negative breast cancer, downregulation of AKT3 significantly inhibits the growth of cancer cells. ${ }^{27}$ AKT3 is also involved in modulating drug sensitivity. ${ }^{28}$ In PCa, knockdown of the AKT family inhibits the growth of cancer cells in vitro and in vivo, suggesting that targeting the AKT family could be an effective treatment for $\mathrm{PCa} .{ }^{29}$ The regulation of the miR-29 family on cell proliferation and apoptosis is also dependent on the $\mathrm{PI} 3 \mathrm{~K} / \mathrm{AKT}$ pathway in acute myeloid leukemia. ${ }^{30}$ In multiple myeloma, the $\mathrm{PI} 3 \mathrm{~K} / \mathrm{AKT}$ pathway is a negative regulator of miR-29b by reducing AKT phosphorylation. ${ }^{17}$ All these previous studies have implicated that AKT3 is a crucial protein in the cellular processes of malignant tumors, including cell growth and drug sensitivity. AKT3 is also involved in the regulation of miR-29b in many cancer types. In our research, we have confirmed for the first time in PCa that miR-29b exerts its tumor-suppressive effects by targeting AKT3. This indicates that the PI3K/AKT pathway is implicated in the miR-29b regulation of $\mathrm{PCa}$.

However, there are some limitations in our study. For instance, the small cohort of $\mathrm{PCa}$ patients and no study on the effects of miR-29b on the CRPC process or androgen receptor (AR) pathway were limitations. To our knowledge, there has been no research exploring the interaction of miR-29b with the AR pathway to date. However, interaction between AR pathways and PI3K/AKT was identified by a recently published study. Its findings suggest a novel mechanism in which AR signaling is involved in PI3K/AKT pathway repression. ${ }^{31}$ Studies have also shown that the regulation of DNMT activity was dependent on AR, suggesting that AR is a key regulator of DNMT expression. ${ }^{32,33}$ In combination with our findings and recently published studies, we will focus further study on the association of miR-29b with AR, DNMTs, and the PI3K/AKT pathway.

\section{Conclusion}

In summary, our findings demonstrate that miR-29b was downregulated in $\mathrm{PCa}$ and was also downregulated in an androgen-independent PCa cell line compared with an androgen-dependent cell line, suggesting its potential role in the CRPC process. miR-29b can inhibit cell proliferation and invasion and enhance cell apoptosis and chemosensitivity of PCa. Furthermore, we have shown for the first time that miR-29b exerts its tumor-suppressive role through involvement in epigenetic regulation and the PI3K/AKT pathway. Further studies with larger samples are needed to elucidate the effects and mechanism of miR-29b in the CRPC process and chemoresistant cancer.

\section{Acknowledgment}

The study was supported by the Science and Technology Agency of Hunan Province in 2013 (2013FJ3055).

\section{Author contributions}

All authors contributed toward data analysis, drafting and revising the paper and agree to be accountable for all aspects of the work. 


\section{Disclosure}

The authors report no conflicts of interest in this work.

\section{References}

1. Siegel R, Naishadham D, Jemal A. Cancer statistics, 2013. CA Cancer J Clin. 2013;63(1):11-30.

2. Wang Y, Nangia-Makker P, Balan V, Hogan V, Raz A. Calpain activation through galectin-3 inhibition sensitizes prostate cancer cells to cisplatin treatment. Cell Death Dis. 2010;1:e101.

3. Bartel DP. MicroRNAs: target recognition and regulatory functions. Cell. 2009;136(2):215-233.

4. Auyeung VC, Ulitsky I, McGeary SE, Bartel DP. Beyond secondary structure: primary-sequence determinants license pri-miRNA hairpins for processing. Cell. 2013;152(4):844-858.

5. Pillai RS, Bhattacharyya SN, Filipowicz W. Repression of protein synthesis by miRNAs: how many mechanisms? Trends Cell Biol.2007; 17(3):118-126.

6. Slack FJ, Weidhaas JB. MicroRNA in cancer prognosis. $N$ Engl J Med. 2008;359(25):2720-2722.

7. Filipowicz W, Bhattacharyya SN, Sonenberg N. Mechanisms of posttranscriptional regulation by microRNAs: are the answers in sight? Nat Rev Genet. 2008;9(2):102-114.

8. Han YC, Park CY, Bhagat G, et al. MicroRNA-29a induces aberrant self-renewal capacity in hematopoietic progenitors, biased myeloid development, and acute myeloid leukemia. J Exp Med. 2010;207(3): 475-489.

9. Nguyen T, Kuo C, Nicholl MB, et al. Downregulation of microRNA-29c is associated with hypermethylation of tumor-related genes and disease outcome in cutaneous melanoma. Epigenetics. 2011;6(3):388-394.

10. Ru P, Steele R, Newhall P, Phillips NJ, Toth K, Ray RB. miRNA-29b suppresses prostate cancer metastasis by regulating epithelial-mesenchymal transition signaling. Mol Cancer Ther. 2012;11(5):1166-1173.

11. Dai F, Zhang Y, Zhu X, Shan N, Chen Y. The anti-chemoresistant effect and mechanism of MUC1 aptamer-miR-29b chimera in ovarian cancer Gynecol Oncol. 2013;131(2):451-459.

12. Steele R, Mott JL, Ray RB. MBP-1 upregulates miR-29b that represses Mcl-1, collagens, and matrix-metalloproteinase-2 in prostate cancer cells. Genes Cancer. 2010;1(4):381-387.

13. Mims A, Walker AR, Huang X, et al. Increased anti-leukemic activity of decitabine via AR-42-induced upregulation of miR-29b: a novel epigenetic-targeting approach in acute myeloid leukemia. Leukemia. 2013;27(4):871-878.

14. Pennati M, Lopergolo A, Profumo V, et al. miR-205 impairs the autophagic flux and enhances cisplatin cytotoxicity in castration-resistant prostate cancer cells. Biochem Pharmacol. 2014;87(4):579-597.

15. Yang SM, Huang C, Li XF, Yu MZ, He Y, Li J. miR-21 confers cisplatin resistance in gastric cancer cells by regulating PTEN. Toxicology. 2013 306:162-168.

16. Zhao G, Cai C, Yang T, et al. MicroRNA-221 induces cell survival and cisplatin resistance through $\mathrm{PI} 3 \mathrm{~K} /$ Akt pathway in human osteosarcoma. PLoS One. 2013;8(1):e53906.

17. Amodio N, Di Martino MT, Foresta U, et al. miR-29b sensitizes multiple myeloma cells to bortezomib-induced apoptosis through the activation of a feedback loop with the transcription factor Sp1. Cell Death Dis. 2012;3:e436.

OncoTargets and Therapy

\section{Publish your work in this journal}

OncoTargets and Therapy is an international, peer-reviewed, open access journal focusing on the pathological basis of all cancers, potential targets for therapy and treatment protocols employed to improve the management of cancer patients. The journal also focuses on the impact of management programs and new therapeutic agents and protocols on
18. Zhang YK, Wang H, Leng Y, et al. Overexpression of microRNA-29b induces apoptosis of multiple myeloma cells through down regulating Mcl-1. Biochem Biophys Res Commun. 2011;414(1):233-239.

19. Wang Y, Zhang X, Li H, Yu J, Ren X. The role of miRNA-29 family in cancer. Eur J Cell Biol. 2013;92(3):123-128.

20. Garzon R, Liu S, Fabbri M, et al. MicroRNA-29b induces global DNA hypomethylation and tumor suppressor gene reexpression in acute myeloid leukemia by targeting directly DNMT3A and 3B and indirectly DNMT1. Blood. 2009;113(25):6411-6418.

21. Amodio N, Leotta M, Bellizzi D, et al. DNA-demethylating and antitumor activity of synthetic miR-29b mimics in multiple myeloma. Oncotarget. 2012;3(10):1246-1258.

22. Pandey M, Sultana S, Gupta KP. Involvement of epigenetics and microRNA-29b in the urethane induced inception and establishment of mouse lung tumors. Exp Mol Pathol. 2014;96(1):61-70.

23. Gravina GL, Ranieri G, Muzi P, et al. Increased levels of DNA methyltransferases are associated with the tumorigenic capacity of prostate cancer cells. Oncol Rep. 2013;29(3):1189-1195.

24. Agarwal S, Amin KS, Jagadeesh S, et al. Mahanine restores RASSF1A expression by down-regulating DNMT1 and DNMT3B in prostate cancer cells. Mol Cancer. 2013;12(1):99.

25. Majumder PK, Sellers WR. Akt-regulated pathways in prostate cancer. Oncogene. 2005;24(50):7465-7474

26. Virtakoivu R, Pellinen T, Rantala JK, Perala M, Ivaska J. Distinct roles of AKT isoforms in regulating $\beta 1$-integrin activity, migration, and invasion in prostate cancer. Mol Biol Cell. 2012;23(17):3357-3369.

27. Chin YR, Yoshida T, Marusyk A, Beck AH, Polyak K, Toker A. Targeting Akt3 signaling in triple-negative breast cancer. Cancer Res. 2014; 74(3):964-973.

28. Yamamoto Y, Yoshioka Y, Minoura K, et al. An integrative genomic analysis revealed the relevance of microRNA and gene expression for drugresistance in human breast cancer cells. Mol Cancer. 2011;10:135.

29. Sasaki T, Nakashiro K, Tanaka H, et al. Knockdown of Akt isoforms by RNA silencing suppresses the growth of human prostate cancer cells in vitro and in vivo. Biochem Biophys Res Commun. 2010;399(1):79-83.

30. Gong JN, Yu J, Lin HS, et al. The role, mechanism and potentially therapeutic application of microRNA-29 family in acute myeloid leukemia. Cell Death Differ. 2014;21(1):100-112.

31. Lee SH, Johnson D, Luong R, Sun Z. Crosstalking between androgen and PI3K/AKT signaling pathways in prostate cancer cells. J Biol Chem. Epub 2014 Dec 19.

32. Gravina GL, Marampon F, Piccolella M, et al. Hormonal therapy promotes hormone-resistant phenotype by increasing DNMT activity and expression in prostate cancer models. Endocrinology. 2011;152(12): $4550-4561$.

33. Chu M, Chang Y, Li P, Guo Y, Zhang K, Gao W. Androgen receptor is negatively correlated with the methylation-mediated transcriptional repression of miR-375 in human prostate cancer cells. Oncol Rep. 2014; 31(1):34-40.

34. Fabbri M, Garzon R, Cimmino A, et al. MicroRNA-29 family reverts aberrant methylation in lung cancer by targeting DNA methyltransferases 3A and 3B. Proc Natl Acad Sci U S A. 2007;104(40):15805-15810.

35. Wang J, Chu ES, Chen HY, et al. MicroRNA-29b prevents liver fibrosis by attenuating hepatic stellate cell activation and inducing apoptosis through targeting PI3K/AKT pathway. Oncotarget. Epub 2014 Oct 22.

\section{Dovepress}

patient perspectives such as quality of life, adherence and satisfaction. The manuscript management system is completely online and includes a very quick and fair peer-review system, which is all easy to use. Visit http://www.dovepress.com/testimonials.php to read real quotes from published authors. 